\title{
Comparative judgments of animal intelligence and pleasantness
}

\author{
ALLAN PAIVIO and MARC MARSCHARK \\ University of Western Ontario, London, Ontario N6A 5C2, Canada
}

\begin{abstract}
Symbolic comparisons of animal intelligence and pleasantness were investigated in five experiments using words and pictures as stimuli. Symbolic distance effects occurred in all experiments. Picture-word effects depended on the experimental design. Mean intelligence comparison reaction time (RT) did not differ for pictures and words when the pairs were presented to independent groups. With repeated measures, mean RT was faster for pictures than for words, but only on the second block of trials. This involved an asymmetrical transfer effect, so that word comparisons on the first trial block greatly facilitated picture comparisons on the second block, whereas picture comparisons did not facilitate subsequent word comparisons. Pleasantness comparisons showed a similar asymmetrical transfer pattern but differed from intelligence comparisons in that the mean RTs were faster with pictures than with words, even with an infinite item set and without prior exposure to the other class of material. The results are discussed in terms of dual coding and other models of symbolic comparisons.
\end{abstract}

This study sought to resolve a theoretical controversy concerning the symbolic processes involved in comparative judgments of abstract attributes of concrete things. Attention focused on animal intelligence, which is abstract in the sense that values on the dimension are not correlated with specific perceptual at tributes such as size, shape, or color. Pleasantness was introduced into the study so that the generality of the initial findings could be tested with another abstract attribute. A central issue here is whether the major aspects of task performance can be interpreted in terms of a dual coding theory of cognition, as opposed to theories that assume a different data base for symbolic comparisons.

Various effects involving comparisons on concrete dimensions, such as size, have been predicted from dual coding theory (Paivio, 1975, 1978a). The basic assumption of the theory is that memory and thought involve the activity of independent but interconnected verbal and nonverbal symbolic systems, which are differentially accessible to verbal and nonverbal stimuli and have different structural and functional characteristics. The verbal system is more directly accessed and activated by linguistic stimuli, such as printed words, whereas the nonverbal system is activated more directly by nonverbal stimuli, such as pictures. The representational units and structures of the nonverbal system presumably "contain" information that is analogous to perceptual information in the sense that there is a continuous, analog relation between the long-term memory information activated by symbolic stimuli, such as names, and the perceptual information aroused directly by the perceptual objects

This research was supported by Grant A0087 from the National Research Council of Canada. Requests for reprints should be sent to Allan Paivio, Department of Psychology, University of Western Ontario, London, Ontario N6A 5C2 Canada. themselves. The perceptual nature of the symbolic information is characteristically reflected in the conscious experience of imagery, so it is appropriate to refer to. the system as a whole as an imagery or image-generating system. At the same time, it is explicitly assumed that the imagery system can be functionally active without necessarily spilling over into reportable imagery.

Dual coding theory provides the following interpretation of performance on symbolic comparison tasks. Verbal stimuli such as animal names (e.g., Moyer, 1973) first activate representations in the verbal system. These in turn arouse symbolic representations in the image system, which are compared with respect to the relevant attributes, such as size. The theory accounts for the symbolic distance effect (faster reaction times, RTs, as differences on the dimension increase) in much the same way as other analog approaches to mental representation (e.g., Moyer, 1973; Moyer \& Bayer, 1976). More pertinent in the present context, it predicts faster RTs for pictures than for words even when the pictures do not directly represent perceptual differences on the relevant dimension. Thus, memory size comparisons are faster for object pairs presented as pictures (equated on depicted size but not on real-life size) than for pairs presented as printed words. This finding is consistent with the assumption that the perceptual memory representations ("imagens") that contain the concrete information are activated more directly by pictures than by words. Also in accord with the theory are the findings that subjects predominantly report using imagery in the performance of such comparisons and that RT correlates with individual differences in imagery ability, as inferred from spatial manipulation tests, but not verbal ability (e.g., Paivio, 1978a).

The dual coding model has been challenged by findings from studies involving comparisons on abstract 
dimensions (e.g., Banks \& Flora, 1977; Holyoak \& Walker, 1976; Kerst \& Howard, 1977). The experiment by Banks and Flora (1977) is most relevant here. They presented subjects with pairs of names and pictures of animals and asked them to choose the member of each pair that was "smarter" (or "dumber"). They found that comparison time was faster for pictures than for words and concluded that this contradicted the dual coding assumption that faster comparisons with pictures means that the processing was mediated by representations in the imagery system. Because the attribute is abstract, the comparison would have to be based on verbal representations, with the result that comparisons should have been faster with words than with pictures or, at least, pictures and words should not have differed in their effect. Since pictures turned out to be superior, Banks and Flora concluded that the finding is more consistent with their semantic coding hypothesis than with dual coding, given the additional assumption that this abstract (essentially linguistic) coding system is accessed more quickly through pictures than through words.

An alternative proposal (Paivio, 1978c) is that, rather than being inconsistent with dual coding theory, such findings call into question the psychological interpretation of attribute abstraction. The general argument is that attributes like intelligence are primarily characteristics of things, or they are based on learned reactions to things. To the extent this is true, subjects in a comparison task would have to access the memory representations of the referent objects before they could make the required judgment. The faster comparison time obtained by Banks and Flora (1977) for pictures than for words is consistent with the interpretation. So, too, are analogous results obtained by Paivio (1978c) for comparisons of pleasantness and monetary value. Subjects in the latter study also reported predominant use of imagery in performing the task, and individuals scoring high on imagery ability had faster comparison times than those with low ability in the case of both pleasantness and value dimensions, and with either pictures or words as items. Verbal fluency showed no significant relation to comparison time, although it emerged as a significant factor in interaction with imagery in the case of pleasantness comparisons. All of the findings appeared to be consistent with the dual coding interpretation: Although abstract, pleasantness and value are nonetheless acquired characteristics of things, and RT will be facilitated by any factor that makes the nonverbal memory representations more accessible.

The implications of the above analysis in regard to individual differences in cognitive abilities have not been directly investigated in the intelligence comparison task. If the theoretical interpretation is correct, faster comparison times should be obtained with pictures than with words (replicating Banks and Flora, 1977), and those who score high on imagery ability tests should have faster comparison times than those who score low. By the same logic, verbal abilities should correlate with comparison time to the extent that performance is dependent on verbal processes.

We tested the above predictions in Experiment 1 by replicating the essentials of the Banks and Flora (1977) experiment using a modified design. Banks and Flora used only eight animals, ranked for intelligence, and divided these into two sets consisting of the four "smartest" and four "dumbest" animals. Within each set, pairs were constructed by pairing each item with every other one and presenting the entire set a number of times. In addition, each subject received blocks of both pictures and word pairs, in counterbalanced order. We used a much larger number of items arranged into independent pairs, and different groups of subjects received pictures and words. Following the experimental task, subjects completed a battery of individual difference tests. To our surprise, we failed initially to replicate Banks and Flora's picture-word effect, and the correlational data involving individual difference variables differed from those obtained with comparisons on other abstract attributes. Experiments 2-5 (the last two involving pleasantness comparisons) sought to resolve these discrepancies, as well as to test a particular theoretical interpretation of the unexpected findings.

\section{EXPERIMENT 1}

\section{Method}

Item pool and normative rating procedure. A pool of 78 animal names was compiled. All animals in the list were easily portrayed in simple line drawings and were familiar to college students. The animals were randomly divided into three lists of 26 each, and these lists were then reproduced for use in a normative rating task. Each of 45 introductory psychology students, participating to satisfy a course requirement, received a test booklet containing a page of instructions, followed by the three lists in random order, and ending with a questionnaire concerning possible strategies, including imagery, that might have been used in the rating tasks. Intelligence was rated on a 9-point scale, on which a rating of 1 denoted lowest intelligence and 9 denoted highest intelligence. Subjects performed the rating task in groups of 4 to 12 and were allowed as much time as necessary to complete the task. Upon completion, subjects filled out the strategy questionnaire. Four specific strategies were included: (1) "Comparing the animals listed with some standard animal or animals not necessarily presented in the list," (2) general verbal strategies (e.g., pronouncing the names of the animals), (3) general use of visual imagery (e.g., simply forming images of the animals), and (4) forming images "of the animals performing some particular behavior suggestive of intelligence." The frequency of use of each of these strategies was rated on a 5-point scale ranging from "used infrequently or never" to "used with all or almost all of the animals listed." In addition, an open-ended question asked subjects to describe any other strategy they might have used and to elaborate on their preferred strategy.

Analysis of the questionnaires revealed a strong and significant preference for the imaginal strategies (all ps $<.005$, except the "general imagery" vs. "comparison with standard" case, $p=.07$ ). Answers to the open-ended question suggested that the second imaginal strategy was the most helpful, as subjects reported imaging the animals in a variety of situations that reflected intelligence. Most subjects also indicated that "com- 
parison with standard" strategy involved the use of visual imagery. ${ }^{1}$

Stimulus materials. Mean intelligence ratings were calculated for each animal; these ranged from 1.49 (worm) to 8.16 (monkey). Two sets of 45 different pairs each were then constructed such that, for 15 pairs in each set, there was an arithmetic difference of approximately 1 between the mean intelligence ratings of the two members; likewise, 15 pairs had arithmetic differences of approximately 3 , and 15 pairs had arithmetic differences of approximately 5. Repetition of individual items was avoided as much as possible, although several appeared more than once in order to obtain equal sets at the three levels of arithmetic difference.

The 90 pairs were then randomly compiled into four different presentation lists. List 1 contained the 45 pairs of one set, followed by the remaining 45 pairs. List 2 contained the two sets in the opposite order. Lists 3 and 4 contained the same order of 90 pairs as in Lists 1 and 2, respectively, but the left-right order of each pair was reversed. Two versions of each list were then constructed, one containing the names of the animals and the other containing line drawings. All pairs were placed on $10 \times 15 \mathrm{~cm}$ cards. Word pairs were typed in IBM Letter Gothic capitals with their midpoints approximately $7 \mathrm{~cm}$ apart; the pictures were all drawn in black ink, subtending approximately the same area $\left(8-9 \mathrm{~cm}^{2}\right)$ and were also placed $7 \mathrm{~cm}$ apart. All lists were balanced so that the more intelligent animal was on the left in half of the pairs and on the right in the other half.

Individual difference tests. Imagery ability was measured by three spatial manipulation (figural transformation) tests. These were space relations (Form A) of the Differential Aptitude Test battery (Bennett, Seashore, \& Wesman, 1947), a shortened version (Linkert \& Quasha, 1941) of the Minnesota Paper Form Board (MPFB), and block visualization (Guilford, 1967). These three tests were selected because they are objective and have been shown to load on a common figural transformational ability factor (Guilford, 1967). They were also moderately intercorrelated in a cumulative sample of subjects that has participated in a series of mental comparison experiments (see Paivio, 1978a, 1978c).

Verbal ability was measured by three tests. One was an associative fluency test that requires subjects to write as many different associations as they can in a $1-\mathrm{min}$ period to each of four concrete and four abstract nouns (see Paivio, 1971, pp. 495496). A second measure of verbal ability was a word beginnings and endings test (adapted from the first- and last-letters tests of L. L. Thurstone). This test requires subjects to write down, in a $3-$ min period, as many words as they can that begin with one letter and end with another. The final verbal measure was an inference test (Guilford, 1967) in which subjects are given one or two statements (premises) followed by various conclusions that could be drawn from them. The task is to decide which of the conclusions can be drawn from the statements without assuming any thing in addition to the information given.

All subjects also received the Full-Range Picture Vocabulary Test (Ammons \& Ammons, 1948). This was initially included because it is purported to be a "quick" test of intelligence. However, the test also appeared to be specifically relevant to the present purposes because it requires transfer from pictorial to verbal information.

Design and Procedure. Forty-eight subjects were drawn from the same population as those in the normative task; 24 received picture stimuli, and 24 received word stimuli. Six people were assigned to each of the four lists within a condition. Subjects were told that pairs of animals (pictures or words) would be presented tachistoscopically, and they were to decide which was the more intelligent member of each pair. Following a ready signal, a fixation point appeared, remained for $1 \mathrm{sec}$, and was immediately followed by a stimulus pair that remained visible for $4 \mathrm{sec}$. The subjects responded by pushing a button with either the right or left thumb, corresponding to whether the more intelligent of the two animals was on the right or the left. RTs and responses were recorded by a PDP- 12 computer in an adjoining room. Between responses, subjects kept their thumbs resting on the buttons in order to facilitate rapid as well as accurate responding. Each subject received 10 practice trials followed by two blocks of 45 trials each. A 2 - to 3 -min rest period between test blocks was optional. This portion of the experiment lasted approximately 20 to $25 \mathrm{~min}$, after which subjects were given the picture vocabulary test. All subjects returned to the laboratory 1 to 4 days later for group administration of the remainder of the individual differences test battery.

\section{Results and Discussion}

Reaction time and error rates. Errors accounted for $4 \%$ and $3 \%$ of the responses in the picture and word conditions, respectively. Mean RTs for correct decisions were computed for each subject for each of the three levels of arithmetic difference. Both mean RT and number of errors were then analyzed by a 2 (pictures vs. words) by 2 (lists) by 3 (symbolic distance) analysis of variance in which difference was a within-subjects factor. The only significant effect, in both analyses, was that of distance $[\mathrm{Fs}(2,80) \geqslant 177.66$, ps $<.001]$. As can be seen in Figure 1, mean RT decreased as the difference in animal intelligence increased. This function was completely consistent with previous findings, but the absence of any picture-word difference was not.

Individual differences. Subject's scores on the individual differences battery were examined through correlational analyses. Table 1 shows, for each test, the Pearson $r$ with comparison mean RT for the total group, as well as for pictures and word groups separately. It can be seen that the only significant predictor of RT for the complete sample was the picture vocabulary test $(\mathrm{p}<.05, \mathrm{r}=-.29)$. The separate analyses showed further that picture vocabulary was the only significant

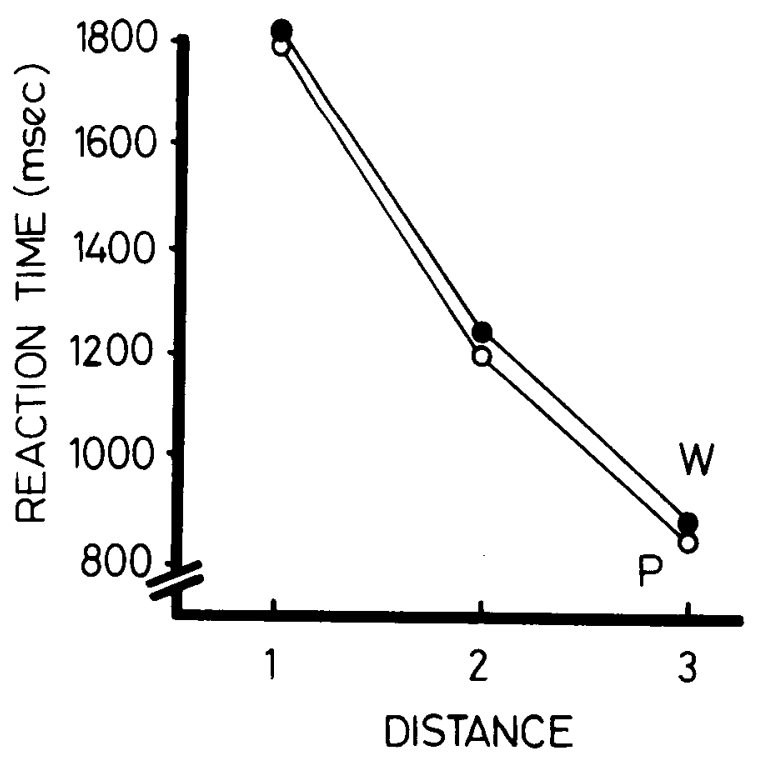

Figure 1. Intelligence comparison mean RT in Experiment 1 for picture pairs and word pairs as a function of pair difference in rated intelligence. 
Table 1

Correlations Between Individual Difference Test Scores and Mean Reaction Time

\begin{tabular}{lrrr}
\multicolumn{1}{c}{ Test } & $\begin{array}{r}\text { Picture } \\
\text { Group }\end{array}$ & $\begin{array}{r}\text { Word } \\
\text { Group }\end{array}$ & $\begin{array}{c}\text { Complete } \\
\text { Sample }\end{array}$ \\
\hline Space Relations & -.32 & .15 & -.07 \\
MPFB & -.08 & .32 & .12 \\
Block Visualization & -.15 & -.01 & -.08 \\
Associative Fluency & -.18 & -.25 & -.22 \\
Word Beginnings and Endings & -.18 & .14 & -.04 \\
Inference Test & -.19 & -.26 & -.21 \\
Picture Vocabulary & $-.57^{*}$ & -.03 & $-.29 \dagger$ \\
\hline
\end{tabular}

${ }^{*} p<.01 . \quad+p<.05$.

predictor for the subjects who received picture stimuli $(\mathrm{p}<.005, \mathrm{r}=-.57)$, but it did not predict comparison mean RT for the word group $(r=-.03)$. In fact, there were no significant predictors of RT for subjects receiving word stimuli. One trivial possibility is that word mean RT is simply less reliable than picture mean RT. This was checked by randomly separating the individual pair RTs from each of the distances into two sets and computing two mean RT scores for each subject. The split-half reliability coefficients, corrected by the Spearman-Brown formula, were .85 for pictures and .95 for words. Thus reliability is actually higher for word mean RT, and we can reject the reliability interpretation.

The overall pattern of correlations differs from those obtained in previous studies, in which the spatial tests predicted performance in comparison tasks involving abstract dimensions (Paivio, 1978c) as well as concrete ones (e.g., Paivio, 1978a). The most that can be said here is that one imagery test, space relations, showed a correlational trend in the appropriate (negative) direction with intelligence comparison time.

The general importance of the results is that they are not easily explained by current theories of mental comparisons. In regard to dual coding, they do not point to the predominance of either imagery or verbal processes in comparisons of intelligence, although complex imagery strategies were apparently involved in the normative intelligence rating task. The absence of a picture-word effect also fails to replicate Banks and Flora (1977) and is, therefore, theoretically inconsistent with their post hoc addition to the semantic coding model, which states that the common, semantic coding system is more quickly accessed through pictorial than through verbal stimuli.

The results of the picture vocabulary test suggest two possibilities. One is that this particular comparison task depends heavily on some general intellectual ability, which is measured by the vocabulary test. The other is that comparison time is influenced by the more specific picture naming ability measured by the vocabulary test. The results are more consistent with the latter than with the former interpretation. If general intelligence were the more important factor, the picture vocabulary test should have correlated with comparison mean RT involving words, as well as pictures, as items. Moreover, one would have expected the inference test to correlate with performance on the comparison task, since that test involves an abstract intellectual skill, namely, syllogistic reasoning. Since the inference test was not predictive and since the picture vocabulary test correlated only with comparison time when pictures served as items, it is more reasonable to conclude that the important factor was related to the subject's ability to translate pictorial information into a verbal code. In terms of dual coding, this would be interpreted to mean that task performance depended on the interconnections between imaginal and verbal systems, rather than on either class of representations alone. Experiment 2 tested an implication of this analysis, which also provided a possible basis for resolv. ing the empirical discrepancy between the present results and those obtained by Banks and Flora (1977) in regard to picture-word effects.

\section{EXPERIMENT 2}

Our Experiment 1 differed from Banks and Flora's (1977) Experiment 2 in two principal ways. First, we presented pictures and words to different groups of subjects, whereas Banks and Flora used a within-groups design in which participants received blocks of trials with one type of material, then a series of trials with the other type. Second, we used an infinite set involving a relatively large number of pairs, whereas Banks and Flora used a small, finite pair set. Either factor may have been responsible for the different results. Experiment 2 accordingly replicated Banks and Flora in terms of both items and general design. We anticipated that this should produce the overall picture-word difference reported by Banks and Flora, but it also permitted separate analyses of the first and second blocks of trials (not reported by Banks and Flora). The analysis of the first trial block would involve independent groups receiving pictures and words. If pictures turned out to be superior to words on that block, it would suggest that the difference between our results and those of Banks and Flora was due to the particular list of items or the finite set design used by the latter. The image-verbal code translation hypothesis suggested the alternative possibility that pictures would be superior to words only (or mainly) on the second trial block, after participants had been exposed to words on the first block. Prior exposure to the words would make the name code highly available when the same pairs are subsequently presented as pictures. The subjects receiving pictures on the first trial block would not have the advantage of augmented verbal code availability.

The experiment required only a small number of subjects and involved a complex repeated-measures design with respect to the picture-word variable. Accord- 
ingly, it was inappropriate for the systematic study of individual differences, and that aspect is not pursued further in relation to Experiment 2 or the subsequent ones. $^{2}$

\section{Method}

Design and Procedure. The stimulus items were the eight animal concepts used by Banks and Flora (1977, Experiment 2). The rank ordering of these animals on intelligence was the same in both Banks and Flora's and our normative rating tasks. In order of decreasing intelligence, the animals were ape, dog, cat, horse, cow, sheep, chicken, and fish.

The eight animals were divided into the four most intelligent and the four least intelligent. Stimulus pairs for the judgment task were created by combining animals only within each category; thus there were six different pairs in each intelligence level. Two different, randomly ordered lists of the 12 pairs were constructed, balancing the number of times a more intelligent animal appeared on the right or the left. Two additional lists contained the same ordering but with the left-right order of each pair reversed. The lists were then reproduced in both picture and word forms as in Experiment 1, creating a total of 96 stimulus pairs ( 12 pairs by 2 lists by 2 left-right orders by 2 forms).

Prior to testing, half of the subjects were shown a list of the pictures and names of the animals, rank ordered on intelligence (cf. Banks \& Flora, 1977, Experiment 2). The remaining subjects received no prior exposure to the animals (no-preview condition). This manipulation allowed us to determine whether Banks and Flora's results were dependent upon their giving subjects previews of the ordered stimuli. Half of the subjects in each of these groups received a block of $\mathbf{4 8}$ picture trials, followed by $\mathbf{4 8}$ word trials; the other subjects received the two blocks in the reverse order.

The testing procedure was identical to that of Experiment 1, except that the subjects received five practice trials with picture pairs before the block of picture trials and five practice trials with word pairs before the word block. The experiment lasted 30-35 min.

Subjects. Twenty subjects were drawn from the same population as those in Experiment 1 and were randomly assigned to the various conditions on the basis of their order of appearance at the laboratory.

\section{Results and Discussion}

Errors accounted for $8 \%$ and $11 \%$ of the responses to picture and word stimuli, respectively, in the nopreview condition, and $6 \%$ to each stimulus type in the preview condition. The error data were analyzed by 2 (preview or no preview) by 2 (material-picture or word) by 2 (order-picture-word or word-picture) by 3 (distance) analyses of variance in which material and distance were within-subjects factors. This analysis yielded a significant main effect only for distance $[F(2,32)=34.13, p<.001]$, so that errors decreased as the rank-order difference increased. The only significant interaction was Preview by Material $[F(1,16)=4.91$, $\mathrm{p}<.05]$.

Mean RTs for correct decisions were computed for each subject for picture and word trials at each level of symbolic distance, that is, at the three rank-order differences $(1,2$, and 3$)$ between pair members. Mean RTs were then analyzed by a 2 (preview or no preview) by 2 (picture or word) by 2 (picture-word vs. wordpicture order) by 4 (trials within each type of material) by 3 (distance) analysis of variance, in which subjects were nested in preview condition and order, and repetitions were nested in material. Significant main effects were obtained for order $[F(1,16)=5.46, p<.05]$, with mean RTs being faster for the word-picture group; material $[F(1,16)=5.68, p<.05]$, with mean RTs to pictures faster than those to words; trials $[F(6,96)=$ $13.06, \mathrm{p}<.0001]$; and distance $[\mathrm{F}(2,32)=40.95$, $\mathrm{p}<.0001]$. The only significant interactions were Material by Order $[\mathrm{F}(1,16)=6.75, \mathrm{p}<.05]$ and Order by Trial $[F(6,96)=2.44, p<.05]$. The former interaction is shown in Figure 2, in which it can be seen that mean RT is lower for pictures than for words only in the case of the group that first compared words and then pictures. The picture-word group showed essentially no effect. Looked at another way, mean RT was slightly faster for words than for pictures on the first trial block and much faster for pictures than for words on the second block. Note, too, that picture mean RTs were much faster on the second than on the first block, but word mean RTs showed a trend in the reverse direction. The simplest factual interpretation of the interaction is that pictures facilitated comparisons given prior expo: sure to their names but not otherwise. The pattern can also be interpreted as an asymmetrical transfer effect: Positive transfer occurred from words to pictures but not from pictures to words.

The Order by Trial interaction resulted from a steeper decline in RT over trials for word-picture than for picture-word subjects. The effect is attributable entirely to differences in the second block, in which subjects receiving pictures showed a progressive decrease in mean RTs and those receiving words progressively increased over the first three trials. The difference is a detailed reflection of the asymmetrical transfer effect already

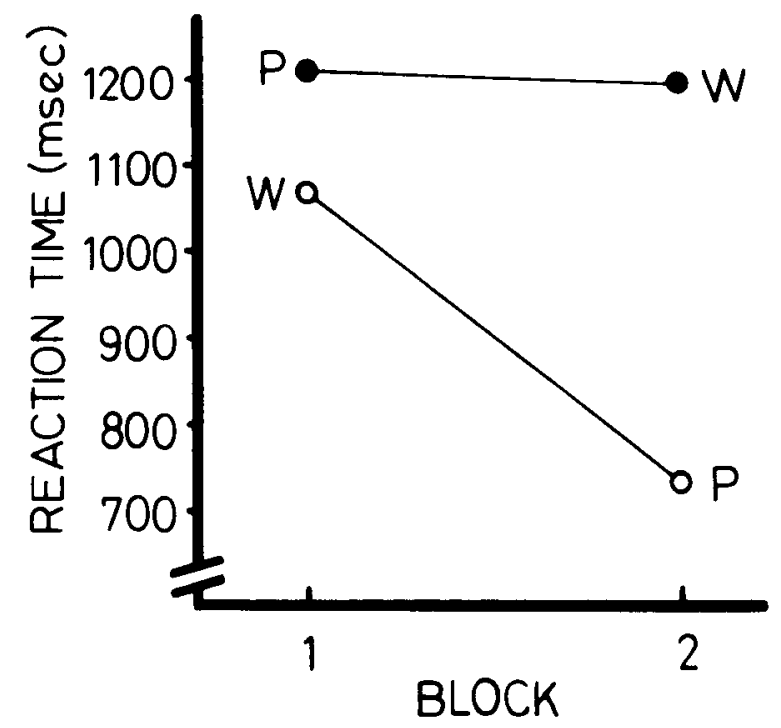

Figure 2. Interaction effect of type of material (pictures vs. words) and trial block on intelligence comparison mean RT in Experiment 2. 
noted. It could not emerge as a triple interaction because of the nested design involved in the experiment.

\section{EXPERIMENT 3}

Experiment 3 was a close replication of Experiment 2, designed to ensure that the results of the latter would generalize to an independent set of items.

\section{Method}

Eight animals were selected from the item pool described in Experiment 1. These were different from, but had mean intelligence ratings as close as possible to, those of the eight animals used by Banks and Flora (1977, Experiment 2). In order of decreasing intelligence, the animals were monkey, porpoise, leopard, raccoon, donkey, rooster, hippopotamus, and turtle. The experimental design and procedure were identical to those of Experiment 2 except that, prior to testing, all subjects were given a preview of the animals' pictures and names, rank ordered by intelligence.

Ten subjects were drawn from the same population of Experiments 1 and 2 and were randomly assigned to the pictureword and word-picture conditions.

\section{Results and Discussion}

Errors accounted for $.8 \%$ and $.3 \%$ of the responses on picture and word trials, respectively. Analysis of the error data yielded a significant main effect only for distance $[F(2,16)=4.04, p<.05]$, resulting from a decrease in errors as rank-order difference increased, and a significant interaction of Material by Order $[F(1,8)=$ $9.68, \mathrm{p}<.025]$. The word-picture and picture-word groups had error rates of .06 and .14 , respectively, on Block 1, whereas the two groups did not differ on Block 2 (.03 and .01, respectively).

As in Experiment 2, mean RTs for correct decisions were computed for each subject and analyzed by a 2 (order) by 2 (material) by 4 (trial) by 3 (distance) analysis of variance in which subjects were nested in order and trials were nested in material. Significant main effects were obtained for material $[F(1,8)=24.10$, $\mathrm{p}<.005]$, trials $[\mathrm{F}(6,48)=5.23, \mathrm{p}<.001]$, and distance $[F(2,16)=21.63, p<.0001]$. Significant interactions were obtained for Material by Order $[F(1,8)=$ $9.04, p<.025]$ and Distance by Trial $[F(12,96)=2.69$, $p<.005]$. Figure 3 depicts the Material by Order interaction. The pattern is similar to that obtained in Experiment 2, insofar as there was no significant pictureword difference for Block 1 trials (between subjects), by a Newman-Keuls test, whereas picture judgments (by word-picture subjects) were significantly faster than word judgments (by picture-word subjects) in Block 2 $(p<.05)$. Viewed in terms of transfer effects, positive transfer again occurred from word to picture trials but not for the reverse order. The Distance by Trial interaction resulted from the symbolic distance effect's being attenuated for the first trial in each block (Trials 1 and 5). The distance effect was strongest (i.e., steepest) for the last trial of each block (Trials 4 and 8 ), and all other trials yielded typical distance effects falling between these two extremes.

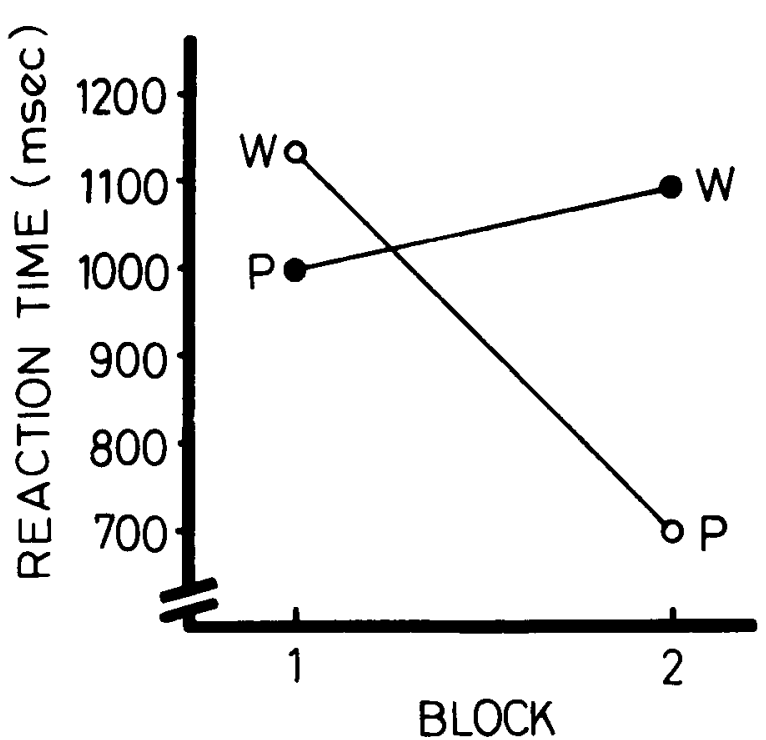

Figure 3. Material by Order interaction effect on intelligence comparison mean RT in Experiment 3.

The picture-word results of Experiments 1-3 seem to be inconsistent with previous findings obtained by Paivio (1978c) for the abstract dimensions of pleasantness and value, both of which involved faster comparisons with pictures than with words as stimuli. Moreover, unlike the intelligence comparison results in Experiment 1 , both pleasantness and value comparisons were significantly faster for subjects who scored high on tests of imagery ability than for those who scored low on such tests. Since the pleasantness comparisons in the Paivio (1978c) study involved an infinite stimulus set, whereas the value comparisons involved a finite set, the differences observed for intelligence comparisons cannot be readily attributed to that procedural feature. However, the pleasantness experiment involved only inanimate objects. Perhaps the results differed because of the difference in stimulus class. Experiments 4 and 5 explored that possibility using pleasantness comparisons with animal concepts. Pleasantness was chosen because it seems as reasonable to compare animals on pleasantness as on intelligence. Attention centered on the effects of pictures as compared with words and the order in which subjects received the two types of material. Experiment 4 involved an infinite-set paradigm, so that the pattern of results for the first trial block could be compared with the pleasantness results of Paivio (1978c) and the intelligence results of Experiment 1 of the present study. Experiment 5 involved a finite set, thereby permitting comparisons with Experiments 2 and 3 .

\section{EXPERIMENT 4}

\section{Method}

Stimulus material. Animal concepts differing in rated pleasantness were drawn from the pool described by Paivio (1978b, Experiment 1). Two sets of 18 different pairs were constructed so that, for 6 pairs in each set, there was an arithmetic difference 
of approximately .75 between the pleasantness ratings of the two members; likewise, 6 pairs in each set had arithmetic differences of approximately 1.50 , and 6 had arithmetic differences of approximately 2.25. Repetition of particular items was avoided as much as possible, but several appeared more than once in order to obtain equal numbers of pairs at the three levels of arithmetic difference. For the same reason, there were four pairs that appeared in both sets. Two lists of 36 pairs were then constructed. Each was composed of the 18 randomly ordered pairs of a set, followed by a repetition of the same pairs in a different order. The repetitions of any particular pair were separated by at least eight other items. Both lists were then reproduced in both picture and word forms.

Subjects. A total of 34 subjects were drawn from the same population as those in the other experiments and randomly assigned to the various conditions on the basis of their order of appearance at the laboratory. In fact, the total sample was made up of two independent groups, tested 5 months apart by two different experimenters. The possibility of differences between the two groups is evaluated below.

Design and Procedure. The testing procedure was identical to that of Experiment 3 except that subjects responded to the more pleasant, rather than the more intelligent, animal of each pair. Seventeen subjects received each list. Nine of those assigned to one list received 36 pairs in word form followed by the same 36 pairs in picture form. Eight other subjects received two sets in the reverse order. Assignment to the second list was counterbalanced so that, overall, 17 subjects received words followed by pictures and 17 received pictures followed by words. Each subject thus had 72 test trials as well as five materialappropriate practice trials prior to each of the two blocks. Prior to presentation of the picture stimuli, subjects were cautioned to choose the more pleasant of the animals represented by the pictures, not the more pleasant line drawing. The experiment lasted approximately $15 \mathrm{~min}$.

\section{Results and Discussion}

Errors accounted for $9 \%$ of the responses each to picture and word stimuli. Mean RTs for correct decisions were computed for each subject at each level of symbolic (arithmetic) difference, separately for pictures and words, in each of the four set repetitions. The mean RT and error data were then analyzed by 2 (experimenter) by 2 (list) by 2 (order-picture-word or word-picture) by 2 (material--picture or word) by 2 (trials within each type of material) analyses of variance in which trials were nested within material and subjects were nested within experimenter, list, and order. The effect of experimenter was negligible, as neither analysis yielded a significant main effect nor any significant interactions that might qualify the effects of interest. This factor is therefore excluded from further discussion.

The error analysis yielded significant main effects of distance $[F(2,52)=43.13, p<.0001]$ and list $[F(1,26)$ $=4.44, p<.05]$. These were qualified by a significant List by Distance interaction $[F(2,52)=4.77, p<.025]$, which resulted from the fact that one yielded a steeper symbolic distance effect than the other.

The RT analysis yielded significant main effects for trials $[F(2,52)=22.11, p<.001]$ and distance $[F(2,52)$ $=18.49, \mathrm{p}<.001]$. A marginal effect of stimulus material was also obtained $[\mathrm{F}(1,26)=3.46, \mathrm{p}<.075]$, but this was qualified by the significant Material by Order interaction $[F(1,26)=7.04, p<.025]$, shown in Figure 4. It can be seen that mean RTs were faster for

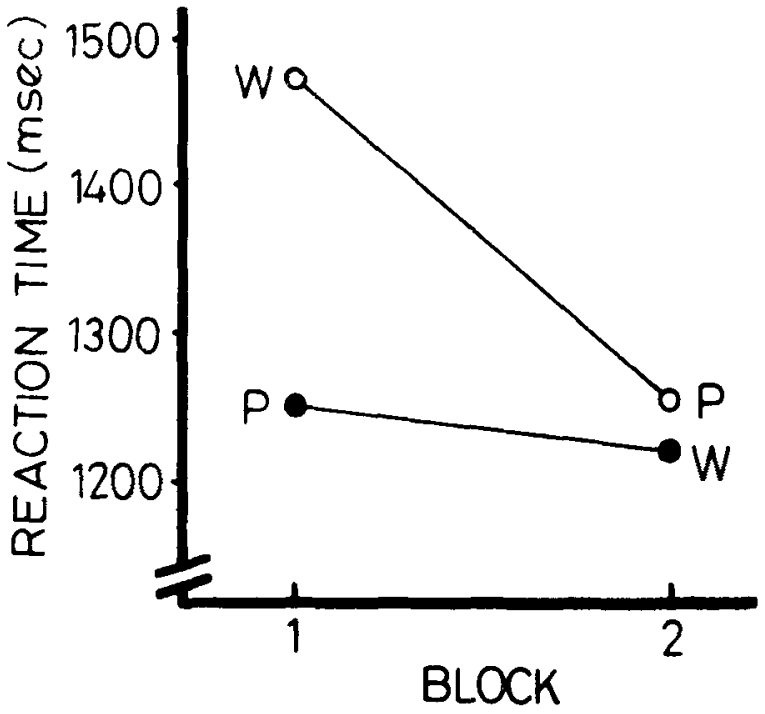

Figure 4. Material by Order interaction effect on pleasantness comparison mean RT in Experiment 4.

picture stimuli than for words on the first block (i.e., between subjects), whereas there was a slight advantage for words on the second block. There were no other significant effects.

These findings replicate those of Paivio (1978b), insofar as comparisons were faster for pictures than for words when there was no prior exposure to the other class of stimulus material. The effect contrasts with the absence of any picture-word difference for intelligence comparisons in the comparable infinite-set conditions of Experiment 1. However, the transfer effects were similar to those observed for intelligence comparisons in Experiments 2 and 3 , in the sense that word comparisons facilitated subsequent picture comparisons of the same concepts but not vice versa. The main difference is that the superiority of pictures over words occurred on the first block of trials in the case of pleasantness and on the second block in the case of intelligence comparisons. Experiment 5 explored the generality of the pleasantness results using a finite-set design.

\section{EXPERIMENT 5}

\section{Method}

Eight animal concepts, differing in rated pleasantness, were drawn from the pool described in Experiment 4. The items were chosen so as to be clearly rank ordered on the dimension, thus eliminating the need for previewing of the ordered list (as in Experiments 2 and 3). In order of decreasing pleasantness, the animals were swan, squirrel, parrot, cow, frog, fly, skunk, and rattlesnake. The experimental design was identical to that of Experiments 2 and 3, except that all subjects received the same random order of the stimulus items. Ten subjects received a block of 48 picture trials followed by a block of 48 word trials, and 10 received the two blocks in reverse order. The procedure was also identical to that of Experiments 2 and 3 , except that there was no preview of the items and that judgments were made on pleasantness rather than on intelligence.

The 20 subjects were all drawn from the same population as in the earlier experiments and were randomly assigned to the 
two conditions on the basis of their order of appearance at the laboratory.

\section{Results and Discussion}

Errors accounted for $1 \%$ of the responses each to picture and word stimuli. Mean RTs for correct decisions were computed for each subject at each level of symbolic (rank-order) difference. The error and mean RT data were than analyzed by 2 (picture-word or wordpicture order) by 2 (picture or word material) by 3 (distance) by 4 (trials within each stimulus form) analyses of variance, in which stimulus material and distance were within-subjects factors.

The error analysis indicated that there were significantly fewer errors to word stimuli than to picture stimuli $[F(1,18)=6.72, p<.05]$ and a robust symbolic distance effect, as errors decreased with increasing distance $[F(2,36)=90.17, p<.0001]$. The only significant interaction was that of Order by Distance by Trials $[F(12,216)=1.92, p<.05]$, which is of no theoretical interest here.

The RT analysis yielded three significant main effects: stimulus materials $[F(1,18)=15.30, p<.005]$, with responses being faster for pictures than words; distance $[F(2,18)=26.39, p<.0001]$, indicating a symbolic distance effect; and trials $[F(6,108)=7.38$, $\mathrm{p}<.0001]$. Significant interactions of Material by Distance $[F(2,36)=4.40, p<.025]$ and Order by Distance by Trials $[F(12,216)=1.87, p<.05]$ were also obtained. The two-way interaction resulted from a reduced picture-word difference at Distance 2. The marginal triple interaction does not qualify any results of interest and will not be considered further.

The critical Material by Order interaction was not significant $[F(1,18)=2.62]$. Nonetheless, as can be seen in Figure 5, the data followed the same general pattern

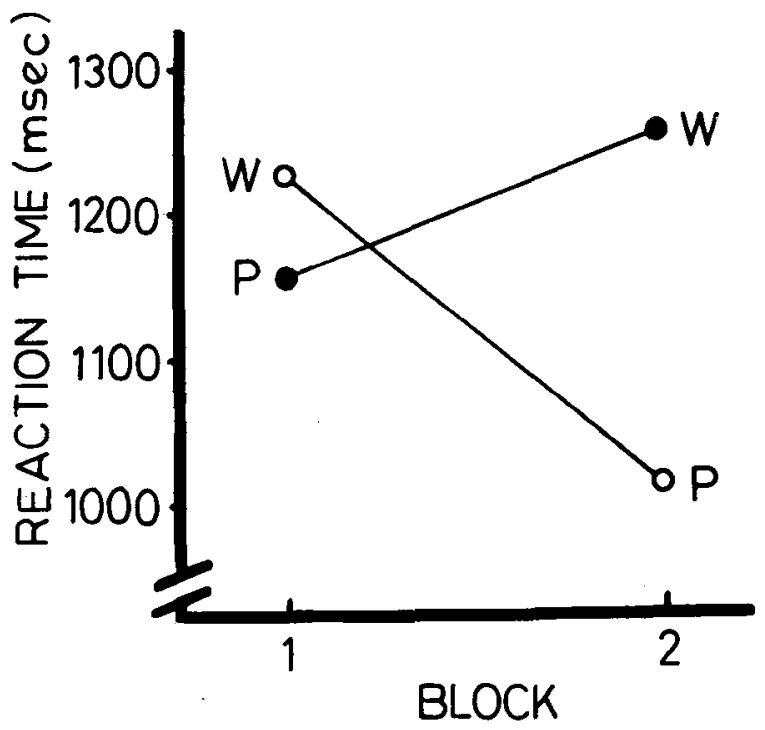

Figure 5. Material by Order interaction effect on pleasantness comparison mean RT in Experiment 5. as intelligence comparisons in Experiment 3 (Figure 3) in regard to the primary locus of the picture-word difference and the asymmetrical transfer effects. The mean RT advantage for pictures was greater in the second than in the first block of trials, and transfer was strongly positive from words to pictures and negative from pictures to words. The effects are unlike those obtained for pleasantness comparisons in Experiment 4, in that, here, comparisons were faster for pictures than for words in the second as well as in the first block. Thus intelligence and pleasantness comparisons share some features in common while differing in others.

\section{GENERAL DISCUSSION}

The discussion deals first with the implications of the results for dual coding and semantic models and then with the more general implications of the asymmetrical picture-word transfer effects observed in Experiments 2-5. Neither the semantic coding nor the dual coding model correctly predicted the results of Experiment 1. To accommodate their finding of faster comparative judgments of intelligence with pictures than with words, Banks and Flora (1977) added the assumption that the abstract semantic coding system, which presumably mediates the judgments, is more quickly accessed by pictures than words. The model would now have to be remodified to account for the observation that pictures are advantageous in this task when preceded by comparisons in which the items are presented as words but not otherwise. It is difficult to see how this could be achieved without a drastic revision of the basic assumptions of the model, unless one simply adds further post hoc assumptions that amount to restatements of various empirical findings themselves. In fact, the asymmetrical transfer effect is incompatible with any theory that assumes a common representational base for pictures and words.

Dual coding theory also failed initially in the sense that previous applications of the theory to comparisons involving other abstract dimensions (Paivio, 1978c) did not generalize directly to animal intelligence. This could mean either that dual coding theory is wrong or that imaginal and verbal processes interact in intelligence judgments in a way that differs somewhat from their contribution to pleasantness and value judgments. The latter interpretation is favored by the results of Experiments 2-5. All experiments showed an interaction of material and trial blocks such that subjects who began with words significantly reduced their reaction times when switched to pictures, whereas those who began with pictures showed no significant overall change on the subsequent word trials, and the trend was sometimes negative. These findings suggest that both pleasantness and intelligence comparisons involve an interaction of imaginal and verbal processes. In addition, however, the image system seemed to dominate more in the case of pleasantness than in the case of intelligence. The latter conclusion follows from the faster pleasantness 
(but not intelligence) mean RTs with pictures than with words even in infinite-set design, where there was no prior exposure to picture labels. It is also consistent with the finding that subjects with high imagery ability, as measured by the spatial manipulation tests, were significantly faster than their low-imagery counterparts in the case of pleasantness (Paivio, 1978c), but imagery ability did not correlate significantly with intelligence comparisons in Experiment 1.

Nonetheless, nonverbal symbolic processes seem to be crucial in intelligence comparisons as well. This conclusion follows from several observations: First, comparison RT became significantly faster when subjects switched from words to pictures, but not vice versa, with the net result that comparisons were sometimes faster with pictures than with words, but never faster with words than with pictures. Second, the picture vocabulary test predicted mean RT in Experiment 1 only when the comparisor task involved pictures as items. Neither observation is consistent with the alternative possibility that linguistic processes predominated in the task. Finally, the correlation of -.32 between space relations test scores and intelligence comparisons mean RT for the picture group in Experiment 1, although nonsignificant, was at least suggestive $(p=.06$, onetailed test) and appropriate in direction.

The data are insufficient to permit a more detailed dual coding interpretation of the intelligence comparison results with high confidence, but several alternatives can be suggested. One possibility is in terms of the additive contribution of verbal and imaginal codes, with the latter carrying more weight in the combined effect (cf. Paivio \& Csapo, 1973). High availability of the verbal code resulting from prior exposure to word pairs or from strong picture naming skill ensures that picture pairs will be appropriately labeled during the comparison task. The congruence of both codes with respect to the concepts being compared results in reliable and speedy responding because both linguistic and world knowledge converge on the problem. To use an extreme example, one "knows" that "chimps" are smart and "worms" are stupid because one has often heard (or read) statements to that effect and because one has observed smart behavior in the former and stupid behavior in the latter. The nonverbal system still carries more weight in the combination because, according to the theory, the referents of the verbal and pictorial signs are represented nonverbally, in the perceptual memory (image) system. Comparisons of the attributes of the referent objects are impossible without access to their symbolic representations. The role of the verbal system is to ensure reliable activation of particular representations as well as to provide access to associated memory information relevant to the comparison task-verbal memories concerning intelligence, nonverbal memories concerning intelligent behaviors (aroused through the referential interconnections between systems), and so on. Subjects in the normative phase of Experiment 1 in fact reported a preference for imaginal strategies when judging intelligence, especially imagining the animals in a variety of situations that reflected intelligence. Whether these involved static images of situations or some kind of active or dynamic imagery (cf. Paivio, 1971, Chapter 2) cannot be clearly inferred from the reports. In any case, the suggested interpretation would be generally consistent with earlier dual coding interpretations of symbolic comparisons (Paivio, 1978c), as well as a variety of other tasks, ranging from free recall (Paivio \& Csapo, 1973) to metaphor comprehension (Paivio, 1979). It is also consistent in principle with the analysis of symbolic comparisons proposed by Kosslyn, Murphy, Bemesderfer, and Feinstein (1977).

An alternative possibility is that the comparisons are essentially based on imagistic representations and that the only contribution of the verbal system is to ensure the arousal of appropriate images. Thus, the picture vocabulary test may be predictive because it taps the subject's ability to identify the things represented pictorially, not because of naming per se. Similarly, prior experimental exposure to word pairs gives the subject practice at generating and comparing imagistic referents of the words. The primed imagery activity is easily re-evoked subsequently by pictures. Initial picture trials, however, provide no practice with image generation to words, hence there is no positive transfer to subsequent word trials.

We cannot choose at this time between the different dual coding interpretations and other possible ones that could be suggested. The important general point is that the findings indicate that intelligence comparisons are highly dependent on the availability of both verbal and nonverbal information, in an interactive manner. Interactions are evident also in pleasantness comparisons, but the dominance of nonverbal processes is more clearly supported by two classes of convergent operations (picture-word differences and individual differences in imagery abilities). Other tasks, such as relative judgments of name frequency appear to be based primarily on the verbal system, as would be expected from dual coding theory (Paivio, 1978b). Thus the intelligence comparison task is somewhat unique in that it seems to be more dependent on the interaction of systems rather than on either one alone.

Finally, we comment briefly on the general implications of the picture-word transfer effect observed in Experiments 2-5. Such effects indicate that situational (episodic) memory as well as semantic memory contribute to task performance. The episodic memory contribution could include any or all of three classes of information related to the first trial block, including (1) memory for the pairs as presented (pictures or words), (2) memory for the representational information aroused from long-term memory by the stimuli (e.g., 
names aroused by pictures, images aroused by words), and (3) memory for the comparison processes or other procedures involved in the task. The first of these is explicitly episodic. The second and third involve episodic information generated from semantic memory during task performance. The extent to which each of these contributed to performance on the second block of trials cannot be ascertained from the present study, but it would be possible to do so by systematically manipulating relevant features (materials, encoding instructions, etc.) of the initial task prior to transferring to a comparison task involving pictures or words as stimuli. The precise nature of the transfer effects is also indeterminate in the present experiments because they did not include the necessary controls for simple repetition effects (i.e., two blocks of trials with words alone or pictures alone). The results permit us only to infer asymmetrical transfer effects that are relatively positive or negative-that is, the word-picture transfer effect was positive relative to the picture-word condition. Conversely, the picture-word transfer effect was negative relative to the word-picture condition. This limitation does not affect the tentative conclusions drawn from the serendipitous results of the present experiments, but more precise conclusions will require a complete transfer paradigm. Systematic experiments of that kind should advance our understanding of the complex process involved in symbolic comparisons generally.

\section{REFERENCES}

Ammons, R. B., \& Ammons, H. S. Full-range picture vocabulary test. Missoula, Mont: Psychological Test Specialists, 1948.

Banks, W. P., \& Flora, J. Semantic and perceptual processes in symbolic comparisons. Journal of Experimental Psychology: Human Perception and Performance, 1977, 3, 278-290.

Bennett, G. K., Seashore, M. G., \& Wesman, A. G. Differential aptitude tests. New York: Psychological Corporation, 1947.

Guilford, J. P. The nature of human intelligence. New York: McGraw-Hill, 1967.

Holyoak, K. J., \& Walker, J. H. Subjective magnitude information in semantic orderings. Journal of Verbal Learning and Verbal Behavior, 1976, 15, 287-299.

Kerst, S. M., \& Howard, J. H., Jr. Mental comparisons for ordered information on abstract and concrete dimensions. Memory \& Cognition, 1977, 5, 227-234.

Kosslyn, S. M., Murphy, G. L., Bemesderfer, M. E., \&
FEinstein, K. J. Category and continuum in mental comparisons. Journal of Experimental Psychology: General, 1977, 106, 341-375.

Like RT, R., \& QuAsha, W. Revised Minnesota paper form board test, Series $A A$. New York: The Psychological Corporation, 1941.

MOYer, R. S. Comparing objects in memory: Evidence suggesting an internal psychophysics. Perception \& Psychophysics, 1973, $13,180-184$.

Moyer, R. S., \& Bayer, R. H. Mental comparisons and the symbolic distance effect. Cognitive Psychology, 1976, 8, 228246.

Paivio, A. Imagery and verbal processes. New York: Holt, Rineharı, \& Winston, 1971.

Paivio, A. Perceptual comparisons through the mind's eye. Memory \& Cognition, 1975, 3, 635-647.

Paivio, A. Comparisons of mental clocks. Journal of Experimental Psychology: Human Perception and Performance, 1978, 4,61-71. (a)

PAivio, A. Dual coding: Theoretical issues and empirical evidence. In J. M. Scandura \& C. J. Brainerd (Eds.), Structural'process models of complex human behavior. Leiden: Nordhoff, 1978. (b)

PAIvio, A. Mental comparisons involving abstract attributes. Memory \& Cognition, 1978, 6, 199-208. (c)

Paivio, A. Psychological processes in the comprehension of metaphor. In A. Ortony (Ed.), Metaphor and thought. New York: Cambridge University Press, 1979.

Paivio, A., \& Csapo, K. Picture superiority in free recall: Imagery or dual coding? Cognitive Psychology, 1973, 5, 176-206.

\section{NOTES}

1. On the basis of the questionnaire data, an upgraded form was constructed. The new version differed from earlier formats in having all strategies stated in general terms and rated on both verbal and imaginal use scales. Previously, each verbal and imaginal strategy has been specifically indicated as a separate item. The data from all three experiments were generally consistent with those of the normative task insofar as subjects preferred strategies involving comparisons of the animals in a variety of intelligence-indicative situations. Unfortunately, the data were not easily interpretable, as subjects seemed unable to distinguish their verbal and imaginal use of the general strategies. Discussion of the questionnaire has therefore been omitted from the three experiments in which it was used.

2. The subjects in Experiments 2 and 3 did in fact complete an individual test battery following the experiment. The resulting correlations between test scores and comparison mean RTs for the different experimental subgroups (with Ns ranging from five to eight), and their pooled combinations over conditions and experiments were all nonsignificant.

(Received for publication May 14, 1979; revision accepted October 8,1979 .) 